\title{
A price to pay for tumor regression
}

\author{
Cell Research (2015) 25:763-764. doi:10.1038/cr.2015.44; published online 21 April 2015
}

Killing cancer cells can have undesired side effects. Upon drug treatment, drug-sensitive cancer cells secrete an array of growth factors that stimulate the proliferation and dissemination of drug-resistant cells in the population.

Acquired resistance to therapy represents a formidable hurdle to the successful treatment of cancer. This is true both for conventional chemotherapeutic regimen and the more recent "targeted" cancer drugs that act selectively on the pathways that are deregulated in cancer. Resistance to targeted agents is often cell-intrinsic through the selection of variants that harbor a secondary mutation that interferes with the drug treatment. However, paracrine signaling has also been shown to play a role in drug resistance. For instance, in melanoma paracrine production of Hepatocyte Growth Factor (HGF) can confer resistance to the selective BRAF inhibitor vemurafenib $[1,2]$. Others have highlighted the role of tumor stroma in induction of epithelial to mesenchymal transition in the cancer cells, which in turn can cause drug resistance. In a recent study, Obenauf and colleagues add a new and unexpected dimension to paracrine drug resistance signaling [3].

In this study, vemurafenib-sensitive and -resistant $B R A F$ mutant melanoma cells were mixed in different ratios and injected into mice. The unexpected finding was that both the proliferation and the rate of metastasis of the drug-resistant cells were enhanced in the presence of drug-sensitive melanoma cells, but only when vemurafenib was administered to the mice. This observation indicates that cancer cells, when exposed to the drug that they are sensitive to, somehow stimulate the proliferation of drug-resistant subclones in the population. This phenomenon was not limited to melanoma, but was also seen in lung cancer cell lines. Moreover, when drugresistant melanoma cells were injected into the bloodstream of mice carrying a drug-sensitive melanoma tumor, the drug-resistant cells were rapidly recruited to the tumor in the presence of drug, showing that the drug-sensitive tumor "attracted" the drug-resistant variants from the bloodstream. This is again consistent with the notion that the regressing drug-sensitive tumor cells produce factors that stimulates the growth of drug-resistant clones of the tumor. Consistent with this, conditioned culture media from drug-treated drug-sensitive cancer cells stimulated the proliferation of drug-resistant clones of the same cancer cells. Consequently, the authors named the secreted activity of the drug-sensitive cancer cells the "Therapy Induced Secretome" (TIS).

To identify the components of this secretome, the authors performed transcriptome analysis of multiple drug-sensitive cells following drug treatment. Key genes that were repeatedly activated included HGF and IGF1, growth factors previously identified involved in resistance to vemurafenib in melanoma [4]. Binding sites for the transcription factor FRA1 (a member of the AP1 family) were enriched in the promoters of genes regulated by drug treatment. FRA1 was inhibited by drug treatment in drug-sensitive cells only and indeed, FRA1 knockdown by RNA interference in melanoma cells produced a growth-accelerating effect on drug-resistant melanoma cells. Further pathway analysis of transcripts induced in drug-resistant cells highlighted the PI3K-AKT pathway as a key mediator of proliferation of drug-resistant cancer cells. Consistent with this, the authors showed that inhibition of the PI3K-AKT pathway with small-molecule drugs reduced the growth-stimulatory effect of the TIS both in vitro and in vivo, providing a potential combination strategy to counter the effects of the TIS [3].

These findings add a new dimension to the already well-established role of paracrine signaling in drug resistance. The secretion of growth factors by drugsensitive tumor cells not only reduces the response of drug-sensitive cells to therapy, but also further enhances the growth and dissemination of any pre-existing drug-resistant subclones in the population (Figure 1). It is well established that receptor tyrosine kinase signaling can lead to reduced responses to targeted cancer drugs of both melanoma and lung cancers [5]. What is new here is that these signals can emanate from the regressing tumor itself, thereby providing a local microenvironment that is conducive for proliferation and metastasis of specific subclones within the regressing tumor population. The findings that tumor cells under stress release soluble factors is not without precedent. For instance, oncogene-induced senescence also leads to secretion of cytokines having paracrine mitogenic effects [6]. The authors show here that blood-borne drug-resistant tumor cells "home in" on the regressing drug-sensitive tumor cells after drug treatment, most likely because this microenvironment is most 


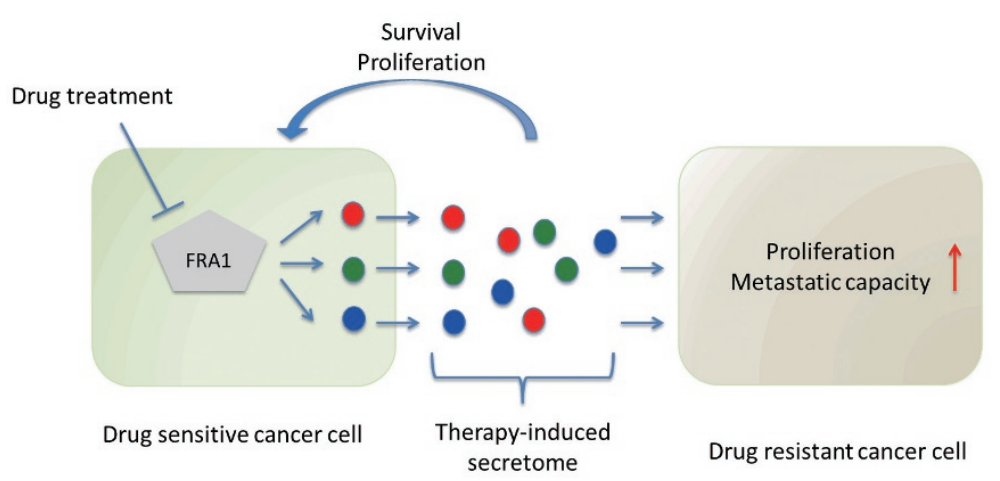

Figure 1 Therapy-induced effects of secreted factors. When drug-sensitive cells are treated with targeted cancer drugs, a program of gene expression is induced through suppression of the FOS-related transcription factor FRA1. This transcriptional program leads to secretion of a number of different growth factors that not only enhance the survival and proliferation of the drug-sensitive cells, but also lead to a marked increase in proliferation and metastatic capability of a pre-existing drugresistant population in the tumor.

advantageous to their proliferation. A similar situation may apply to patients that experience wound-healing responses after cancer surgery. The remaining circulating tumor cells take advantage of the local cytokines released during the wound-healing response to proliferate at that site. For instance, local cancer recurrences at the site of tooth extraction are well-documented [7]. The role of FRA1 in regulating proliferation and metastases may have a cell type-specific component. In the present study, suppression of FRA1 was found to promote induction of the TIS and stimulate growth of drug-resistant cancer cells. However, in other studies, FRA1 was shown to be required for distant metastasis of breast cancer tumor cells [8]. Irrespective of the role of FRA1 in this process, it is clear that oncologists must take these paracrine effects of the regressing tumor into account when treating cancers with targeted agents.
In this respect, the suppression of TIS by inhibitors of PI3K-AKT signaling is encouraging. However, the efficacy of combinations of targeted agents can be limited by toxicity, which limits the possible combinations of targeted agents.

\section{Rene Bernards ${ }^{1}$}

${ }^{1}$ Division of Molecular Carcinogenesis and Cancer Genomics Center Netherlands, The Netherlands Cancer Institute, Plesmanlaan 121, 1066 CX Amsterdam, The Netherlands Correspondence: Rene Bernards Tel: +31-20 5126973

E-mail: r.bernards@nki.nl

\section{References}

1 Straussman R, Morikawa T, Shee K, et al. Nature 2012; 487:500-504.

2 Wilson TR, Fridlyand J, Yan Y, et al. Nature 2012; 487:505-509.

3 Obenauf AC, Zou Y, Ji AL, et al. Nature 2015; 520:368-372.

4 Heynen GJ, Fonfara A, Bernards R. Cell Cycle 2014; 13:3808-3817.

5 Sun C, Bernards R. Trends Biochem Sci 2014; 39:465-474.

6 Kuilman T, Michaloglou C, Vredeveld LC, et al. Cell 2008; 133:1019-1031.

7 Hirshberg A, Leibovich P, Horowitz I, et al. J Oral Maxillofac Surg 1993; 51:13341337.

8 Desmet CJ, Gallenne T, Prieur A, et al. Proc Natl Acad Sci USA 2013; 110:5139-5144. 